\title{
Validation of the new Hipparcos reduction
}

\author{
F. van Leeuwen
}

Institute of Astronomy, Madingley Road, Cambridge, UK

Received 26 July 2007 / Accepted 8 August 2007

\begin{abstract}
Context. A new reduction of the astrometric data as produced by the Hipparcos mission has been published, claiming accuracies for nearly all stars brighter than magnitude $\mathrm{Hp}=8$ to be better, by up to a factor 4 , than in the original catalogue.

Aims. The new Hipparcos astrometric catalogue is checked for the quality of the data and the consistency of the formal errors as well as the possible presence of error correlations. The differences with the earlier publication are explained.

Methods. The internal errors are followed through the reduction process, and the external errors are investigated on the basis of a comparison with radio observations of a small selection of stars, and the distribution of negative parallaxes. Error correlation levels are investigated and the reduction by more than a factor 10 as obtained in the new catalogue is explained.

Results. The formal errors on the parallaxes for the new catalogue are confirmed. The presence of a small amount of additional noise, though unlikely, cannot be ruled out.

Conclusions. The new reduction of the Hipparcos astrometric data provides an improvement by a factor 2.2 in the total weight compared to the catalogue published in 1997, and provides much improved data for a wide range of studies on stellar luminosities and local galactic kinematics.
\end{abstract}

Key words. space vehicles: instruments - methods: data analysis - catalogs - astrometry - instrumentation: miscellaneous

\section{Introduction}

Since the publication of the Hipparcos catalogue (ESA 1997) there have been suggestions, though not always well founded, of the presence of systematic errors in the astrometric data the catalogue contains (see for example: Narayanan \& Gould 1999; Soderblom et al. 2005). Suspicion was in particular raised concerning the reliability of the parallaxes at the sub-milli arcsecond level. Such errors, if present, may become apparent when for example averaging parallax information for open clusters. Here systematic or correlated errors would be the most conspicuous and damaging, as the formal accuracy on a cluster parallax can well exceed those of individual stellar parallax determinations in the catalogue. Some problems with the Hipparcos astrometric data, as due to inaccuracies in the along-scan attitude reconstruction, have since been suggested by Makarov (2002) and identified in detail by van Leeuwen (2005). As these problems were found to be curable, the concept of a new reduction (van Leeuwen \& Fantino 2005) became a viable option. This reduction was recently completed and a full description of the processes used is now in press (van Leeuwen 2007). In that publication, however, the emphasis is on illustrating the proper use of the Hipparcos astrometric data and the way these have been obtained in the new reduction. The data used to illustrate these processes were obtained from (mainly nearly-final) iteration phases. The present paper provides the overall quality check on the new catalogue as it will become available in 2007 through the data disk included in van Leeuwen (2007) and early 2008 through AstroGrid ${ }^{1}$.

With the availability of a new and potentially more accurate astrometric catalogue, it is now also possible to do a detailed investigation of the catalogue published in 1997. The importance of this investigation is that any observed differences need to be

\footnotetext{
${ }^{1}$ http://www2 . astrogrid.org/
}

fully understood from the differences between the old and new reduction methods.

One may get the impression that errors were made in the original reduction of the Hipparcos data, but this is not the correct way of describing what has happened. Several aspects have to be considered before judging the performance of the original reduction by the two consortia, FAST (Kovalevsky et al. 1992) and NDAC (Lindegren et al. 1992):

1. the original aim of the mission was to achieve a parallax accuracy of around 2 milli arcseconds (mas). The final results as published in 1997 were about a factor two better, despite a very serious problem with the orbit of the satellite;

2. computing hardware available around 1993 to 1996 , when the final calculations took place, was much less powerful than what is available today. This reflected in the time required for a single iteration (more than 6 months), and a limitation to how often this could be done. It also limited the possibility to interact efficiently with the reduced data to check results. What took more than 6 months some 12 years ago, takes currently about a week on a single desktop computer;

3 . the Hipparcos measurement principle is complex and sensitive. Some of this was well understood before the start of the mission, not least thanks to the preparatory work of Lennart Lindegren. An input catalogue (Turon et al. 1992) with an even distribution of target stars over the sky was created to ensure a stable astrometric solution for the mission data. How sensitive the solution really is became only clear during the iterations of the new reduction. In particular, the principal requirement of linking the data in the two fields of view is critical, and links can far more easily be weakened than was imagined 25 years ago, when in the 1980s the input catalogue was constructed. 
This paper, and the two associated with it (van Leeuwen 2005; van Leeuwen \& Fantino 2005), are therefore not an accusation of bad workmanship in the creation of the catalogue published in 1997. The intention is rather to show that the principle of the Hipparcos instrument, now also being implemented for the Gaia satellite, is valid and is the only way one may measure directly, fully reliable absolute parallaxes, and do so for large numbers of stars. These papers show that the reduction of the Hipparcos data is far from simple, but also that complications associated with the reduction of those data are now much better understood, and can be sufficiently controlled.

For clarity of the current paper, the main issues that have been resolved in the new reduction, and which have led to a significant improvement in accuracies for the new catalogue are briefly summarized here. For a more comprehensive description the reader is referred to the two papers already mentioned above (van Leeuwen 2005; van Leeuwen \& Fantino 2005) and to van Leeuwen (2007).

The noise on the astrometric data as gathered by Hipparcos originates from two main sources. The first source is the Poisson noise on the original photon counts from which the measurements of transit times were derived, and which sets the accuracy limit for those data. The second is the noise from the along-scan attitude reconstruction, which provides the reference frame used to transform transit times to one-dimensional positions on the sky, a transformation that needs to be free from distortions. Significant improvements in the attitude reconstruction have been obtained in the new reduction through a much improved understanding of peculiarities in the dynamics of the satellite, in particular concerning non-rigid events (scan-phase discontinuities) and hits by dust particles. Abandoning the greatcircle reduction technique (van der Marel 1988; van der Marel \& Petersen 1992) for a global iterative solution allowed for a better control over the attitude reconstruction, but makes it necessary to iterate the reduction between the attitude reconstruction and the reconstruction of the astrometric parameters. A similar iteration is also planned, and has already been tested extensively (O'Mullane et al. 2006), for the Gaia data reductions (Lindegren 2005).

The final improvement for the new reduction comes from a better understanding of the connectivity requirement in the reconstruction of the along-scan attitude. The connectivity requirement, when applied correctly, enables sufficient contributions to the along-scan attitude from both fields of view of the telescope. This is essential for obtaining a reconstructed sky free from local small distortions. Such distortions can ultimately lead to local variations in the parallax zero point. Weights of data contributions from the two fields of view can in practice fluctuate considerably, despite the construction of the Hipparcos Input Catalogue, which was designed to provide a more or less homogeneous distribution of selected stars over the sky (ESA 1992). The weight-ratio allowed in the along-scan attitude reconstruction for the two fields of view affects the convergence of the final catalogue as well as the noise level for the brightest stars. Experiments done during the construction of the new catalogue arrived at an optimal value of around 2.7. A larger value would make the convergence move along very slowly, a smaller value would create unacceptable noise levels for the brightest stars.

The Hipparcos data are obtained in the form of transit times, which are transformed to angular positions along the scan directions. These positions are referred to as asbscissae, and in all applications the input consists of abscissa residuals, the differences between the observed and predicted angular positions along the scan direction. This definition of the abscissae is different from that used in the original reductions, where abscissae referred to angular positions as projected onto a reference great circle. In the new reduction, the abscissae are measured strictly with respect to the instantaneous scan direction.

The present paper will refer to various observational aspects of the Hipparcos mission, without going into detail. Extensive descriptions on how the Hipparcos data were obtained can be found in Volume 3 of ESA (1997), as well as in van Leeuwen (2007).

This paper has been organized as follows. Section 2 summarizes the statistical tests carried out on the internal formal errors at the relevant stages of the data reductions. Also considered are correlation levels of the abscissa residuals and the global dependencies of the formal errors on the astrometric data. Section 3 presents the external verification of the data, though the possibilities for this are rather limited at accuracies better than 0.3 mas. Section 4 gives a brief impression of the potential impact of the new reduction. Finally, Sect. 5 presents a summary of the conclusions derived from this study.

\section{The internal accuracy verification}

\subsection{The transit data}

The positional information obtained by Hipparcos is derived from the modulated signal created by the transit of a stellar image over a regular grid of transparent lines. These transits are examined over fixed time intervals of just over $2 \mathrm{~s}$, and it took 9 such transits to cross the 0.9 field of view. The grid, with a periodicity of 1.2 arcsec as projected on the sky, creates a signal which is dominated by a first and second harmonic. The modulation phases define the transit time for a stellar image with respect to a reference grid line. The modulation amplitudes of the harmonics are weak functions of stellar colour and position on the grid, dependencies which are calibrated and used to detect double stars. Data for different stars are obtained pseudo simultaneously by switching the small sensitive area of the photomultiplier detector between different stellar images as these move across the grid. The first formal error to enter the reduction is therefore the error on the estimate of the modulation phase, represented traditionally by $\beta_{3}$ (for details see van Leeuwen 2007):

$\sigma_{\beta_{3}} \approx \frac{0.25}{M_{1} \sqrt{I_{\mathrm{tot}}}} \operatorname{arcsec}$.

Here $M_{1}$ is the relative modulation amplitude of the first harmonic, with a typical value between 0.6 and 0.75 , mainly depending on the colour index of the observed star. The integrated intensity $I_{\text {tot }}$ is obtained from the total photon count of the signal. The scaling factor 0.25 is stable to within 1 per cent over the mission, and contains amongst others the conversion from modulation phase to positional displacements in the focal plane. Figure 1 shows a histogram of the "normalized" formal errors as observed for the transit data in one orbit near the start of the mission.

The integrated photon count $I_{\text {tot }}$ for an observation varied considerably, as stars from magnitude -1 to 12.5 were observed. In general, the brighter stars were assigned less observing time than the fainter ones, but this provided only a weak compensation for the intensity differences, as the possible maximum ratio in observing time was only a factor 16 (equivalent to $3 \mathrm{mag}$ ), while the typical range was more like a factor four to six. Figure 2 shows an example of the distributions of formal errors near the start of the mission. These diagrams immediately expose a problem with the published data. The formal errors on 


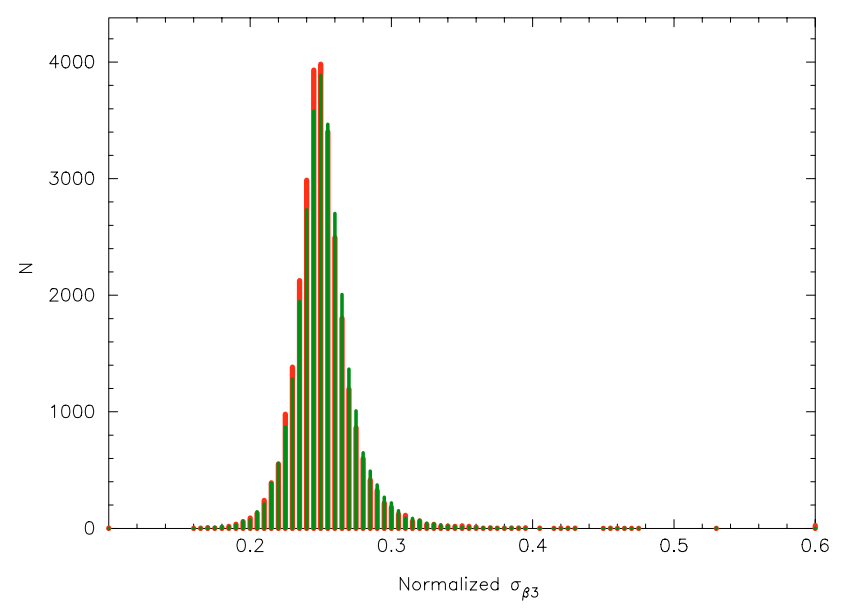

Fig. 1. The normalized errors $\left(\sigma_{\beta_{3}} \times M_{1} \sqrt{I_{\text {tot }}}\right)$ on the modulation phases for transits over the modulating grid. The data are for orbit 174, 21 January 1990.

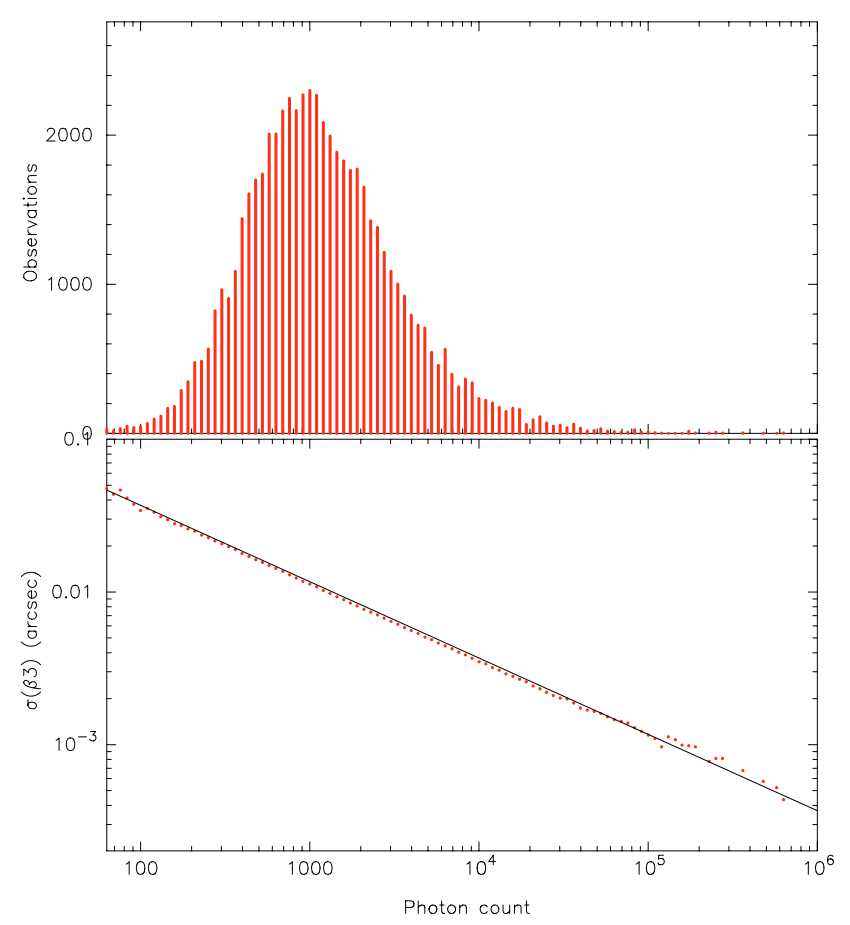

Fig. 2. The distribution of numbers of observations (top) and mean errors on the modulation phase (bottom), as a function of the integrated photon count of the observation. The diagonal line in the lower diagram shows the slope of the photon noise relation. The offset is fairly arbitrary because of variations in $M_{1}$. This also reflects in the variations in the data for the brightest transits. The data are for orbit 174 .

single transits of the brightest stars are already smaller than the accuracy of the reconstructed position as derived from the astrometric parameters and their covariance matrix in the 1997 publication, which are based on all mission data combined. In the old reduction, the noise on the astrometric parameters for these stars was entirely caused by inaccuracies in the attitude reconstruction, and showed significant correlations for in particular bright stars at small separations on the sky (see further Sect. 2.5).

The transit observations are used as input for the along-scan attitude determination. They are combined with a preliminary estimate of the attitude (based on star mapper data) and predicted positions from the last version of the astrometric

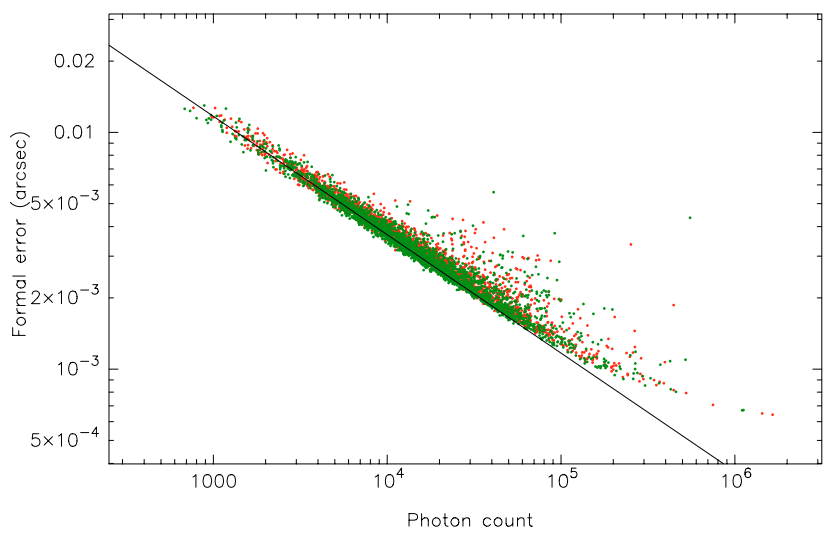

Fig. 3. The formal errors on the combined abscissa residuals, including the errors on the predicted positions. The diagonal line is drawn in the same position as in Fig. 2. The upturn towards the brighter transits reflects the contribution from the uncertainty in the stellar reference positions. The data are for orbit 174 .

catalogue to provide abscissa residuals. Studies concerning the dynamics of Hipparcos (van Leeuwen \& Fantino 2003) have shown that regular positional variations with amplitudes above 0.1 mas are mostly restricted to time scales above $50 \mathrm{~s}$. It therefore seems justified to group data over $10 \mathrm{~s}$ intervals. There have been noted a few instances when still not fully-understood disturbances of the rotational motion of the satellite took place on shorter time scales. This affected only small parts of about half a dozen orbits. For those few data sets, the relevant parameter (the density of nodes in the spline fitting) for the attitude model was adjusted. The grouping of data, which is done for each field of view separately, has a number of advantages. It creates pseudo measurements with smaller formal errors, which allow for a better detection of attitude disturbances such as hits and basic-angle drifts. The number of observations in an otherwise relatively large solution is reduced by about a factor 5, requiring less processing time. The formal errors assigned to individual (pseudo) measurements take into account the accuracies of the predicted transit times as derived from the available astrometric solution. This means that the formal errors tend to be larger than that given by the photon statistics alone, in particular for the brightest stars (Fig. 3). Where more than one observation of the same star contributed to a group, the contribution of the external error was appropriately adjusted.

\subsection{The basic angle and other instrument parameters}

Stability of the basic angle is a critical requirement when it comes to triangularization of the sky with an instrument like Hipparcos. The stability of the basic angle is naturally not exact, and some noise is left from small variations. The presence of such variations, and their scale can be demonstrated by means of a detailed study of the residuals left after the along-scan attitude reconstruction. To make this visible, residuals have been averaged over 16 min intervals (equivalent to a 45 degrees rotation of the satellite), so that the noise level is reduced by nearly a factor 10.

Figure 4 is an example of such an abscissa-residual test, showing a correlation with an amplitude of about 0.4 mas, which would contribute an additional abscissa noise of 0.3 mas. A test involving 10 successive well-covered orbits shows the same approximate level of the correlations, but there are no signs of systematic variations, i.e. the variations are not linked to the rotation 


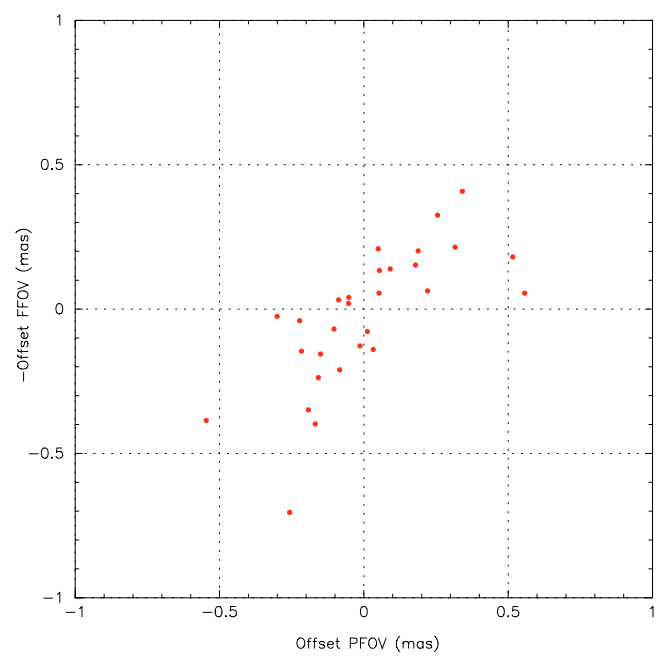

Fig. 4. The correlation between abscissa residuals in the preceding and following fields of view. The residuals have been averaged over intervals of $16 \mathrm{~min}(1 / 8$ rotation of the satellite) to increase the resolution. The correlation shows small variations of the basic angle taking place at time scales longer than 16 minutes. The data are for orbit 174 .

phase or the orbital phase of the satellite. The observed variations are likely to be a reflection of the temperature-control cycle of the payload. As was explained by van Leeuwen (2005), a spinsynchronous modulation could have led to a systematic offset in the parallaxes, but is clearly not observed. That such a modulation is unlikely to be present was also shown indirectly through the determination of the parallax zero point, which was found to be correct to within 0.1 mas (Arenou et al. 1995; Lindegren 1995).

A few actual basic-angle drifts have been observed over the mission, and these are linked to known thermal-control problems of the payload. A detailed description and identification of the orbits affected is found in van Leeuwen (2007).

Next to the basic angle, three sets of instrument parameters have been used to describe the large-, medium- and small-scale distortions of the description of the geometric projection of the sky on the focal plane and the measuring grid. The large-scale distortions describe the projection effects of the telescope optics and their variation with time. These distortions are represented by a two-dimensional third-order polynomial in position and a linear colour correction. The medium-scale distortion corrections reflect the small-scale optical and the grid distortions, which are observed to be very stable over the mission, with a maximum amplitude just below 1 mas. The large- and mediumscale distortions are both resolved over the grid. The small-scale distortions represent the printing characteristics of the modulating grid, and are reconstructed to an accuracy level of approximately 0.1 mas. This correction, too, is very stable over the mission. It is resolved only as a function of the transit ordinate by means of collecting abscissa residuals left after the astrometric solutions, at a resolution of 1000 intervals across scan. There is also a fourth correction, which describes the detailed colour dependence of the abscissa residuals. The detailed colour and the small-scale geometric correction also provide information on local abscissa noise, from which formal error corrections have been derived as a function of transit ordinate and star colour index. These corrections compensate for inaccuracies in the calculation of the modulation factor $M_{1}$ in Eq. (1), when calculating formal errors. Further details on these calibrations are provided by van Leeuwen (2007). For the assessment of the internal accuracies of the Hipparcos data, the most important aspect of these calibrations is that they appear to have been able, together, to represent the geometric distortions down to a level of about 0.1 mas, which is insignificant with respect to other noise contributions.

\subsection{The along-scan attitude}

The along-scan attitude has been derived using the fullydynamic model (FDM) as presented first by van Leeuwen \& Fantino (2005). For details the reader is referred to that paper or to van Leeuwen (2007). Here only a summary relevant to the accuracy assessment is presented.

Through the FDM the underlying torques acting on the satellite are reconstructed. From these torques are derived the satellite rotation rates by means of integration over the Euler equation for the motion of a rigid body in space. Error angles are then obtained through integration of the rotation rates. Both integrations require starting points, which are also part of the attitude modelling. In an iterative fitting procedure for the along-scan attitude, abscissa residuals are fitted as a function of time with a 5 th order exact spline function (van Leeuwen \& Fantino 2003; van Leeuwen 2007), the second derivative of which provides the correction to the torque model. The integrations are carried out over uninterrupted intervals. Interruptions can be caused by thruster firings, scan-phase jumps, (micro meteoroid) hits or large gaps in the data stream (due to occultations, perigee passages, no ground-station coverage etc.). The underlying, continuous, torque model covers time intervals between any two gaps in the data that are longer than $1 \mathrm{~min}$. For each integration interval, the starting values for the pointing and rate integrations are also determined. Thus, the reconstructed attitude can be evaluated at any point of time, except for times very close to the discontinuities.

The attitude-reconstruction process ensures the proper level of connectivity between observations in the two fields of view. This is achieved through controlling the relative weights of data from the two fields of view contributing to the attitude reconstructions. These weights are checked for each interval between nodes of the spline function. A maximum weight ratio of 2.72 is allowed. For any larger ratio, the largest weight is reduced to 2.72 times the smaller weight for the data contained in the node interval. Very few exceptions were made to this rule, to allow some short stretches of time with data from only one field of view.

\subsection{Field-of-view transits and astrometric parameters}

The next step in the processing of the transits is the combination of the abscissa residuals (after correcting for the large- and medium-scale distortions and determining the along-scan attitude) to mean abscissa residuals for field-of-view transits. This may involve up to 10 individual measurements. A goodness-offit statistic is calculated for each field-of-view transit, based on the formal errors of the transit measurements as described above. Figure 5 shows a typical example of the observed distributions for this statistic. There is an indication of a small underestimate of the formal errors on the transit data in this particular case, shown by the offset of the data from the ideal Gaussian distribution. Data of this kind have been collected for all orbits and examined for deviations as part of the quality assurance process.

The formal errors on the field transits are still governed by the same dependencies as for the transit data, and are dominated 


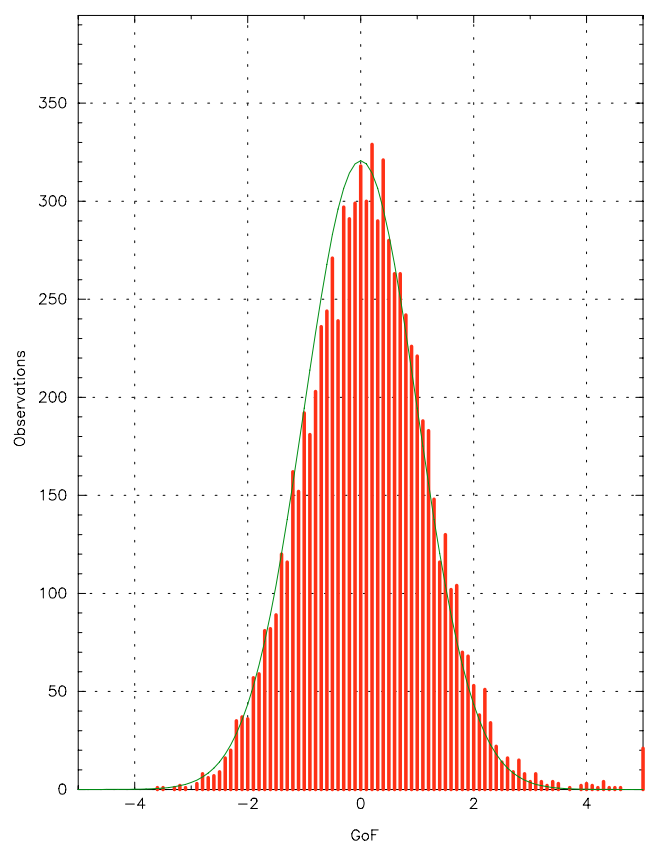

Fig. 5. The goodness-of-fit statistics for the combination of the transit data to field transits. The histogram shows the observed distribution, and the curve the equivalent Gaussian distribution for the same number of observations. The data are for orbit 174 .

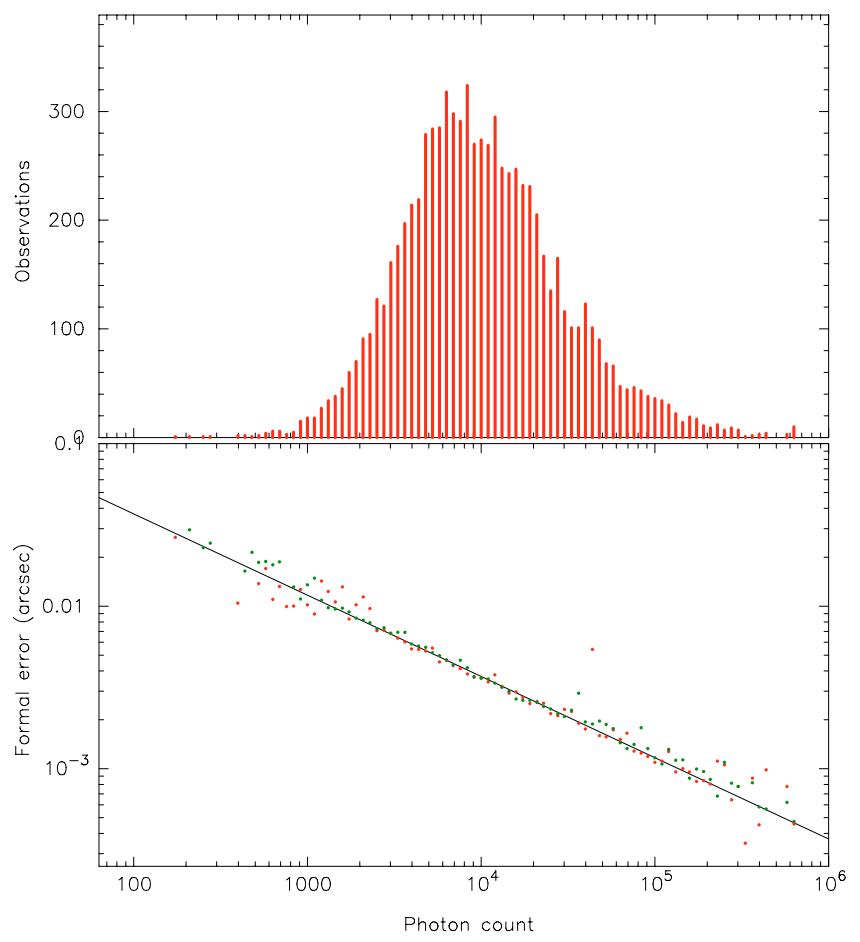

Fig. 6. The distribution of formal errors (bottom) and numbers of observations (top) for the field transits. The position of the diagonal line in the bottom diagram is the same as in the similar Figs. 2 and 3 above.

by photon count statistics, as can be observed in Fig. 6 . These field transit data are the input to the astrometric-parameter estimation and the calibration of the small-scale geometric distortions. They are referred to as field-transit abscissa residuals (FTAR).

At each cycle of the iterative process that ultimately builds the final astrometric catalogue, corrections to the assumed

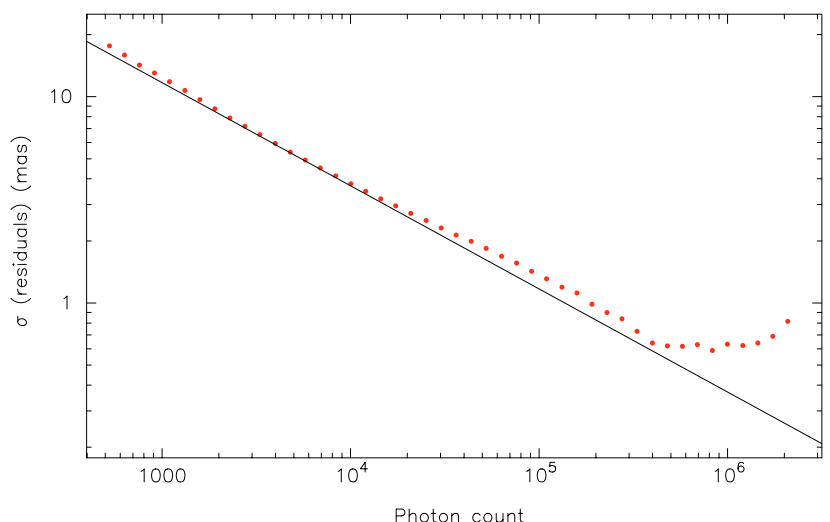

Fig. 7. The dispersions of abscissa residuals after fitting of astrometric parameters for single stars with five-parameter solutions. The diagonal line is the same as in the preceding figures, and shows that these residuals are still largely dominated by photon noise. Only at the brightest end, the attitude noise (at about 0.6 mas) dominates.

astrometric parameters have been determined based on the FTARs. These corrections have been accumulated as corrections to the astrometry in the catalogue of 1997. A basic fiveparameter astrometric solution has been sufficient for application to 102072 out of a total of 117955 stars. Indicative of the improvement in quality of the new solution is the number of stars for which the old catalogue gave a so-called stochastic solution (1561), that are solved as normal solutions in the new reduction (962). Stochastic solutions represent cases where remaining residuals are significantly larger than expected. This can be caused by unresolved orbital motion, but in case of the published data, also by an accidental accumulation of unresolved problems in the satellite attitude reconstruction.

Examination of the dispersions of the FTARs after the astrometric parameter solutions (Fig. 7) provides the final check on the internal consistency of the data, as well as a measure of the remaining attitude noise. This noise is found to be approximately 0.6 mas as applicable to a FTAR. In comparison, the attitude noise left in the 1997 catalogue was 1.5 to 2 mas at the level of the combined FTARs for an orbit. With on average 4.5 FTARs per orbit, the equivalent attitude noise in the original reduction was about 3 to 4 mas, at least a factor five larger than in the new reduction. This reduction in attitude noise reflects in the formal errors of the astrometric parameters, which are determined by photon statistics down to about magnitude 3.5 to 4 in the new reduction, but only down to magnitude 8 to 9 in the 1997 catalogue.

\subsection{Abscissa-residual error correlations}

A rather troublesome issue with the astrometric catalogue published in 1997 was the correlation level of the abscissa residuals, and the way this affected the determination of astrometric parameters for, for example, star clusters (see further van Leeuwen $\&$ Evans 1998). Two kinds of determinations of the correlation levels have been made for the new reduction, for the field transits and for mean orbit transits. The latter serves as material for comparison with the 1997 publication, as abscissa-error correlations for the published data were determined for mean orbit transits rather than for the field transits that are available in the new reduction. As abscissa-error correlations originate from inaccuracies in the along-scan attitude determination, they naturally show most clearly in the data for the brightest transits, where the 


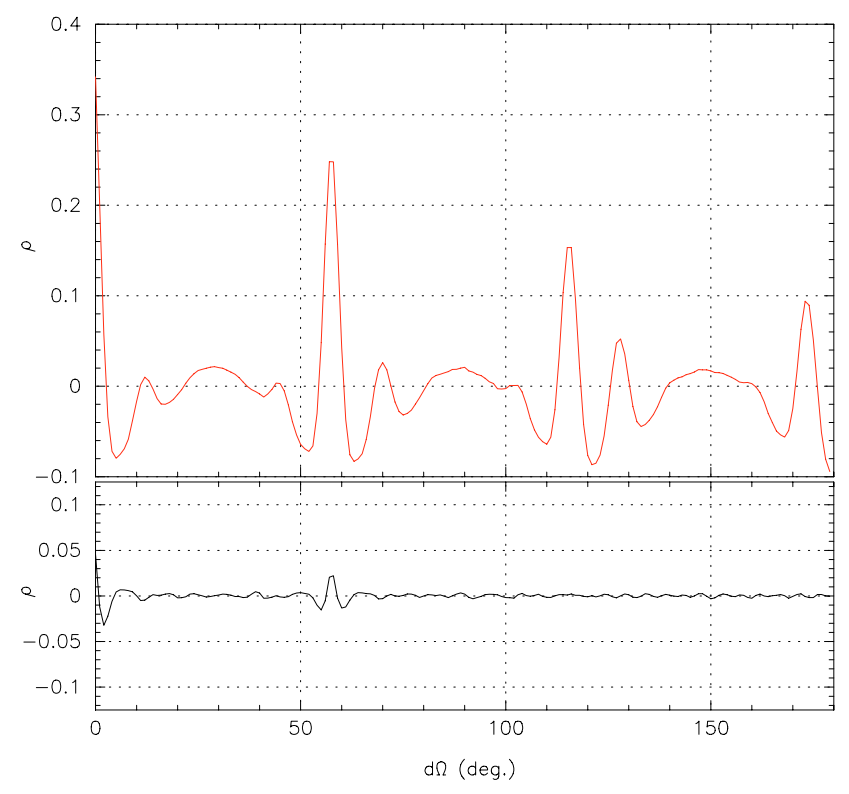

Fig. 8. Abscissa-error correlations for mean orbit transit residuals with formal errors below 3 mas as a function of separation along the reference great circle. Top: the NDAC data as published in 1997; bottom: the new reduction. The peaks are found at intervals of an integer times the basic angle of 58 degrees, and fold back at 180 degrees.

photon noise is lowest. Figure 8 shows for the old and the new reduction the correlations for mean orbit transits as derived for transits with formal accuracies better than 3 mas. This essentially selects only the brighter stars. The large difference between the NDAC results of 1997 and the new reduction is the result of the improved accuracy of the attitude modelling. The repeated peaks for the NDAC data show how the great-circle reduction process used for building the 1997 catalogue was replicating, up to 9 times, local errors in the along-scan attitude solution.

For the processing of the new catalogue data the correlations between the FTARs are relevant rather than those between the mean transit residuals per orbit. To examine these correlations, data on field transit residuals (with formal errors below 6 mas) have been collected over all orbits of the mission. The resulting curve is shown in Fig. 9 (upper graph). The error limit is about equivalent to the one used above for the mean orbit transits.

What is also important for the astrometric data derived from the FTARs is how correlations between abscissa residuals can accumulate into correlations in astrometric parameters. This is largely determined by the coincidence factor, as introduced by van Leeuwen (1999). The coincidence factor for star $A$ with respect to star $B$ gives the fraction of observations of star $A$ for which there are observations of star $B$, contained within the same field of view passage. Also taken into account is the typical length between nodes in the along-scan attitude modelling, which stretches well beyond the width of the field of view. The coincidence factor thus defines to what extent the underlying abscissa-error correlations could accumulate into correlated errors in the astrometric parameters. Coincidence only plays a role for stars at relatively short separations on the sky (Fig. 9, lower graph). Coincidences with specific stars in the other field of view are rarely repeated over the mission. The coincidence level for FTARs is generally about a factor two lower than for mean orbit transits, where apparent coincidence would be related to projection on a reference great circle rather than actual coincidence of the measurements.

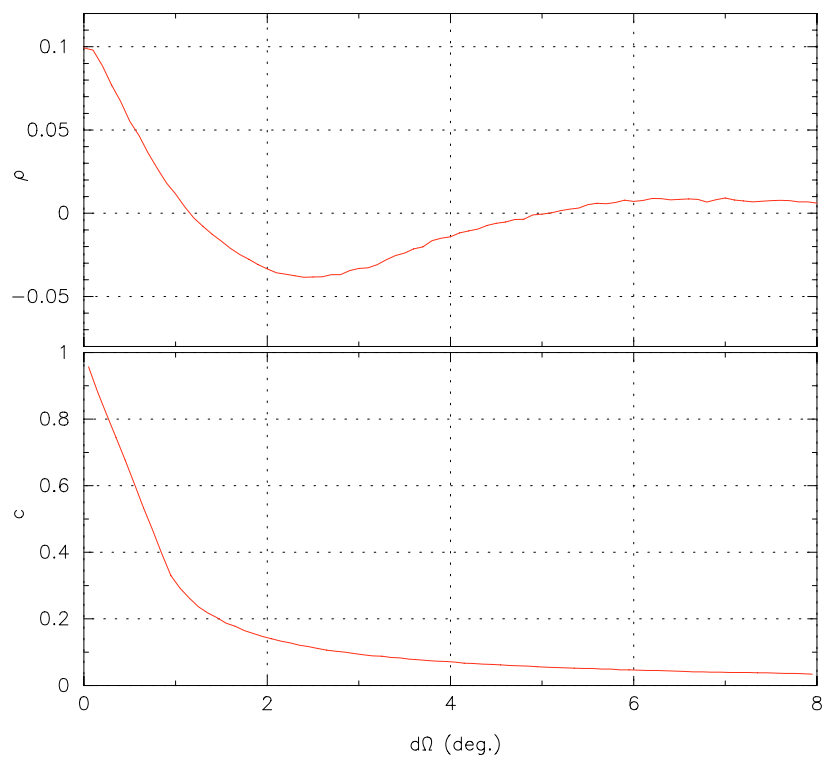

Fig. 9. Top: abscissa-error correlation level $(\rho)$ for field-transit abscissa residuals (FTARs) with formal errors below 6 mas, as a function of separation along the scan direction. Bottom: coincidence fraction $c$ of field-transit measurements as a function of separation on the sky. The coincidence data are based on a random selection covering 10 per cent of all single stars in the new catalogue.

The coincidence factor together with the correlation level determine the chance of accumulating correlated errors in the astrometric parameters; the coincidence fraction defines the average percentage of observations of two stars that could be correlated, at a level defined by the correlation for that separation (or slightly smaller due to projection effects). Considering that the correlation levels in the new reduction are nearly ten times smaller than in the 1997 catalogue, and the coincidence factors two times smaller, it will be clear that for the new reduction the sensitivity to accumulation of abscissa-error correlations into astrometric-parameter correlations has been very significantly reduced.

Considering the smaller formal errors on the astrometric parameters in the new reduction, the much lower correlation levels, and the lower coincidence statistics, the differences between the new and the original astrometric parameters can reveal correlation levels in the published data. An example of such test is shown in Fig. 10 for the parallax data of stars with formal errors on the parallax less than 1.2 mas in the new reduction. The level of the correlations is quite high, but their extent over the sky is small, and agrees, at least qualitatively, with what could be expected based on the coincidence statistics for mean orbit abscissa residuals. Beyond a radius of 3 degrees no correlations are observed, though there may also be (small) anti-correlations existing for stars at 180 degrees separation, considering the abscissaerror-correlation dependencies (Fig. 8) for the 1997 catalogue.

\subsection{Formal errors and their dependencies}

The main error dependencies left in the new catalogue are:

- the photon noise on the observations, which is a function of the magnitude of the star at the time of observation and the (variable) integration time;

- the number and distribution of observations, which is a function mainly of ecliptic latitude, but which has been locally affected by incompleteness in the scan coverage due to lack of 


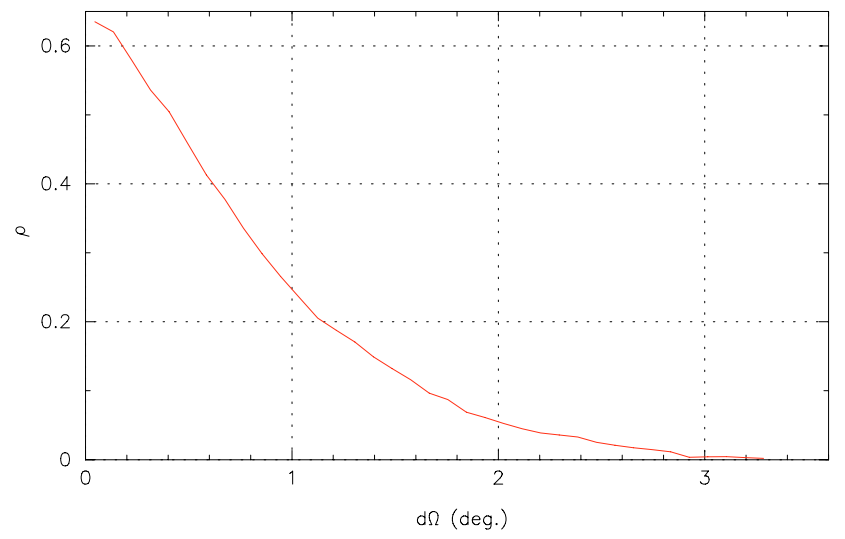

Fig. 10. Parallax-error correlations in the 1997 catalogue, as observed from the correlation level $\rho$ between the parallax updates in the new catalogue for neighbouring stars as a function of their separation on the sky. Only stars with formal errors on the parallax (in the new catalogue) below 1.2 mas were selected. Based on the abscissa-error correlations for the old and the new catalogue, these correlations most likely originate from the 1997 catalogue.

ground-station coverage, observing conditions (like excessively high background signal), and data loss, most of which was due to hardware problems;

- calibration noise, primarily originating from the attitude reconstruction.

All these contributions can be recognized in Fig. 11. The main linear relation represents the photon statistics. The two discrete distributions are due to the differences in coverage between the ecliptic poles (lower band, good coverage) and ecliptic plane (upper band, relatively poor coverage). The photon noise relations do not continue towards the brightest stars, as the errors for these stars are still dominated by attitude noise.

Figure 12 shows the distributions of parallax errors (below 3.6 mas) for 98560 stars with simple five-parameter solutions in the 1997 publication and the new reduction. Some of the discrete features in the distribution for the 1997 catalogue may be due to the a posteriori corrections that had been applied to formal errors on abscissa residuals.

\subsection{Special cases}

For at least two special cases of stars in the Hipparcos catalogue the new reduction has provided a more than average improvement. The first group concerns secondary stars in double systems with separate measurements for the two components, but with the components still sufficiently close to cause disturbed transits. The improvement on those stars stems from the resolution of the data in field transits, which provides much better estimates of the instantaneous orientation of a double system with respect to the modulating grid than can be obtained from mean orbit transits. This, in turn, allows for a significant improvement in the prediction of the position of the brighter component of the system relative to the targeted fainter component. An accurate relative position with respect to target star and the modulating grid is essential for correcting the signal of the target star from the disturbance of the bright companion. An example of how this affected the astrometric data of such systems is shown in Table 1. Contrary to the 1997 solution, the new reduction now clearly shows this system as a physical binary.

The second group of stars are those with extreme red colour indices, many of which are in addition variable. In the new

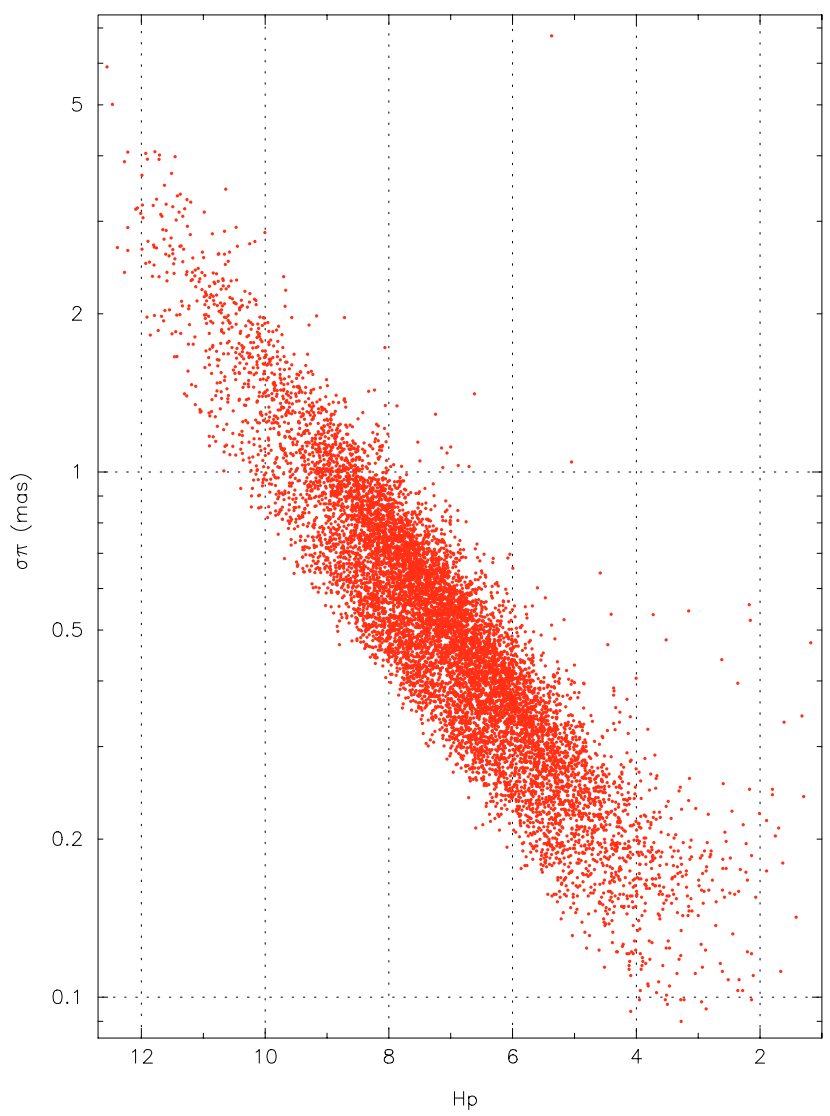

Fig. 11. The formal errors on parallaxes for 9976 stars with relative errors on the parallaxes of less than 5 per cent. The near-linear relation with magnitude represents the photon statistics. The two bands in the distribution represent stars around the ecliptic poles (lower band) and stars around the ecliptic plane (upper band).

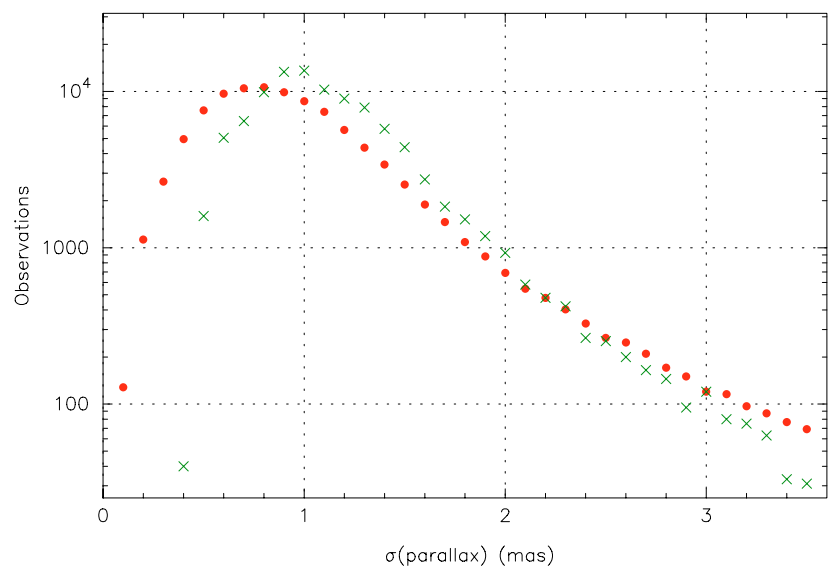

Fig. 12. Histogram of the number of single stars for intervals of width 0.1 mas in parallax error. The dots show the results for the new solution, the crosses for the 1997 solution.

reduction, epoch-resolved colour indices have been used, which has two effects. The calibrations of geometric parameters can also be supported by the very red stars, and in the application of a calibration, the applicable colour of a star is more accurately known. Data on the epoch-resolved colours of the red stars has been provided by Dimitri Pourbaix (see further: Platais et al. 2003; Knapp et al. 2001, 2003). 
Table 1. Published and new determinations for the astrometry of the primary (HIP 71) and secondary (HIP 70) components of a wide-binary system.

\begin{tabular}{llrrrrl}
\hline \hline Star & Param. & Old & $\sigma$ & New & $\sigma$ & units \\
\hline 70 & $\varpi$ & 5.25 & 13.87 & 5.95 & 3.08 & mas \\
71 & $\varpi$ & 9.13 & 1.84 & 7.33 & 1.36 & mas \\
70 & $\mu_{\alpha *}$ & -46.78 & 16.42 & -23.25 & 3.27 & mas/yr \\
71 & $\mu_{\alpha *}$ & -24.50 & 2.05 & -23.61 & 1.39 & mas/yr \\
70 & $\mu_{\delta}$ & -0.88 & 11.24 & -18.58 & 1.92 & mas/yr \\
71 & $\mu_{\delta}$ & -19.47 & 1.40 & -20.55 & 0.85 & mas/yr \\
\hline
\end{tabular}

\subsection{Conclusions on the internal accuracies}

The internal accuracies of the new astrometric data appear to be in all aspects consistent with what should be expected on the basis of the two most important noise contributors, the photon noise and the attitude noise. The reduction in correlation level between abscissa residuals is in agreement with the reduced noise on the attitude, which has been the result of provisions that were made for two major types of disturbance (scan-phase discontinuities and hits) in the attitude modelling.

\section{External accuracy verification}

\subsection{Comparison with radio-star observations}

The final transformation of the Hipparcos catalogue to an inertial reference frame involved several types of measurements, and a complete description can be found in Chapter 18 of Volume 3 of ESA (1997). Here the main interest is on whether there are any significant differences between the 1997 catalogue and the new reduction in as far as these transformations are concerned. The focus is on the VLBI measurements of 12 stars, and the transformations between the astrometric parameters as determined through radio observations, the 1997 catalogue and the new reduction.

In the preparation of the linking of the Hipparcos catalogue to the ICRS as defined by radio observations (Arias et al. 1995; Lestrade et al. 1995, 1999) a dozen stars were measured over a period of time also partly covered by the Hipparcos mission. Four out of these twelve stars are double stars, and may therefore be complicated in their solutions. Some of these stars are in addition variable. The radio-star positions have been measured relative to extra-galactic sources and are thus effectively equivalent to absolute in parallax and proper motion.

The parallax comparison is shown graphically in Fig. 13, distinguishing between single and double stars. The weighted mean of the parallax difference for the 8 single stars is determined as $-0.013 \pm 0.48$ mas, with a unit-weight standard deviation (the square root of the normalized $\chi^{2}$ ) of $1.55 \pm 0.39$. A similarly non-significant result is obtained when also including the double stars. The VLBI and new Hipparcos parallaxes can thus be considered to be in good agreement, with a possible hint of an underestimate of the formal errors in one or both sets of observations.

The transformation in either position or proper motion between two catalogues is given by a set of small rotations $\left(\epsilon_{1}, \epsilon_{2}, \epsilon_{3}\right)$ around three orthogonal axes as:

$\left[\begin{array}{l}\mathrm{d} \alpha_{*} \\ \mathrm{~d} \delta\end{array}\right] \approx\left[\begin{array}{rrr}-\cos \alpha \sin \delta & -\sin \alpha \sin \delta & \cos \delta \\ \sin \alpha & -\cos \alpha & 0\end{array}\right] \cdot\left[\begin{array}{l}\epsilon_{1} \\ \epsilon_{2} \\ \epsilon_{3}\end{array}\right]$.

A derivation of these relations can be found in van Leeuwen (2007). A nearly identical set of equations is used for determining the relative spin between two catalogues, with on the

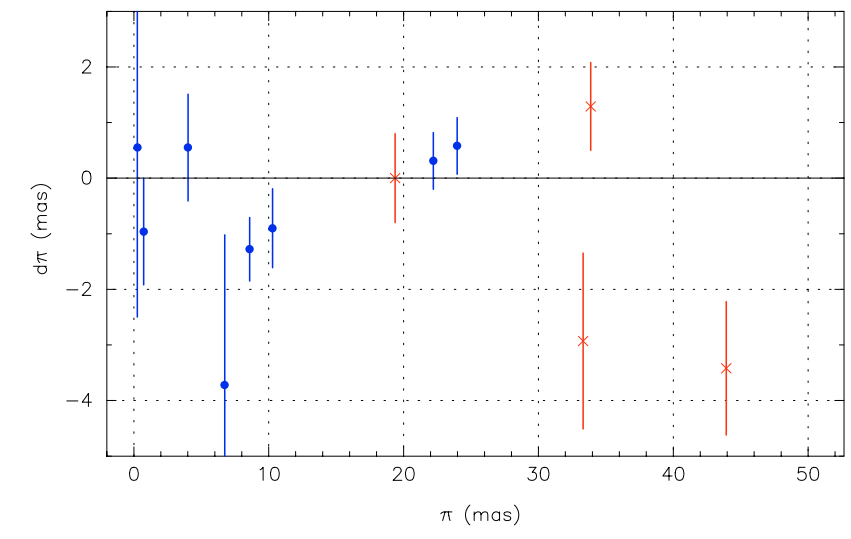

Fig. 13. Differences (VLBI-HIP) in parallax between VLBI measurements and the new reduction, as a function of the parallax. Data points indicated with crosses refer to double stars. Error bars represent the combined formal errors on the two sets of observations.

left-hand side the differences in proper motion and on the righthand side the spin vector $\omega$. A comparison has been made for the proper motions, and is shown in Fig. 14. The differences in proper motion do not represent a significant spin between the two systems. The spin components are determined as:

$\omega_{1}=0.17 \pm 0.36 \operatorname{mas~yr}^{-1}$
$\omega_{2}=0.10 \pm 0.30 \operatorname{mas~yr}^{-1}$
$\omega_{3}=0.40 \pm 0.34 \mathrm{mas} \mathrm{yr}^{-1}$

with a unit-weight standard deviation of 1.8 and 12 observations. Excluding the double stars does not provide an improvement of these results. The comparison in positions (Fig. 15) gives a similar result:

$\epsilon_{1}=-0.11 \pm 0.85$ mas
$\epsilon_{2}=0.06 \pm 0.86$ mas
$\epsilon_{3}=-0.14 \pm 0.79$ mas

with a unit-weight standard error of 2.0. Given the small number of observations, one third of which are double stars, the standard deviations for these transformations are likely to be affected by peculiarities in the astrometric parameters of the individual stars, in particular orbital motion. Differences in the positional origins of the radio and optical signals may also play a role in a few cases.

\subsection{The optical realization of the ICRS}

The Hipparcos catalogue as published in 1997 serves as the optical realization of the International Celestial Reference System or ICRS (Feissel \& Mignard 1998). This section looks at the overall agreement between the new reduction and the 1997 catalogue in positions and proper motions.

The transformations between the new and old catalogues are again based on Eq. (2). The observations are the positional or proper motion differences between the old and the new catalogue. The errors on these observations are partly correlated (when dominated by photon statistics), depending on the brightness of the object. For the brightest stars there is effectively no correlation, and the errors are as given by the 1997 catalogue, while for the faintest stars the correlation can be considerable. With the brighter stars providing most of the weight for the solution, errors have been assigned according to the formal errors given in the published catalogue. The comparisons are based on 


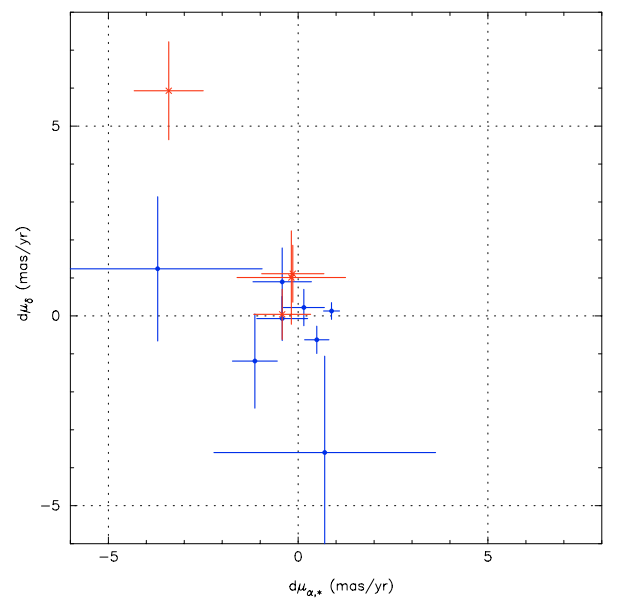

Fig. 14. Differences (VLBI-HIP) in proper motion between VLBI measurements and the new reduction. Data points indicated with crosses refer to double stars. Error bars represent the combined formal errors on the two sets of observations.

the data of 99130 stars with basic five-parameter solutions in both catalogues. In position the following transformation values are found:

$\epsilon_{x}=0.058 \pm 0.002$ mas
$\epsilon_{y}=-0.011 \pm 0.002$ mas
$\epsilon_{z}=0.028 \pm 0.003$ mas,

with a unit-weight standard deviation of 0.87 . As expected, the standard deviation is less than 1.0, reflecting the partial correlation of the data. The same transformation using the formal errors from the new catalogue for calculating the observation weights gives marginally different results, and a unit-weight standard deviation of 1.24. In both cases the rotations observed are, though significant on their own, more than an order of magnitude smaller than the accuracy with which the positions in the published catalogue have been linked to the ICRS to provide its optical realization (Arias et al. 1995; Feissel \& Mignard 1998).

A similar comparison has been made for the proper motions, giving the following values:

$\omega_{x}=-0.001 \pm 0.003 \mathrm{mas} / \mathrm{yr}$

$\omega_{y}=-0.005 \pm 0.003 \mathrm{mas} / \mathrm{yr}$

$\omega_{z}=+0.006 \pm 0.003 \mathrm{mas} / \mathrm{yr}$,

and a unit-weight standard deviation of 0.90 (or 1.26 when new weights are applied). It can therefore be concluded that the new catalogue as a reference frame is essentially identical to the 1997 catalogue, and only very small and insignificant differential rotations have been introduced in its preparation.

\subsection{Parallaxes}

The verification of formal errors on parallaxes in the published catalogue relies primarily on the observed distribution of negative parallaxes. Such verification is only partially possible with the new reduction. The main reason is that amongst those stars for which a negative parallax was derived, the smallest formal error is 0.23 mas. There are no negative parallaxes amongst the 1000 stars with smaller errors than this value, and only two for the first 2400 stars, when ranked according to formal parallax error. Figure 16 shows the overall distribution of negative parallaxes as a function of the formal errors, as well as the histogram

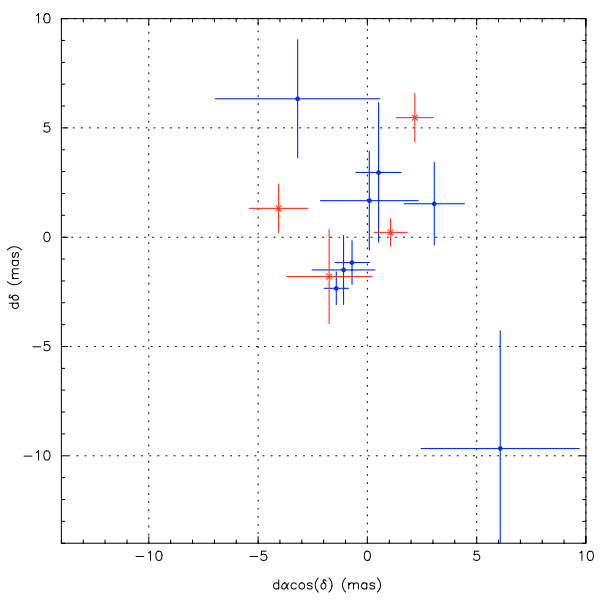

Fig. 15. Differences (VLBI-HIP) in positions between VLBI measurements and the new reduction. Data points indicated with crosses refer to double stars. Error bars represent the combined formal errors on the two sets of observations.

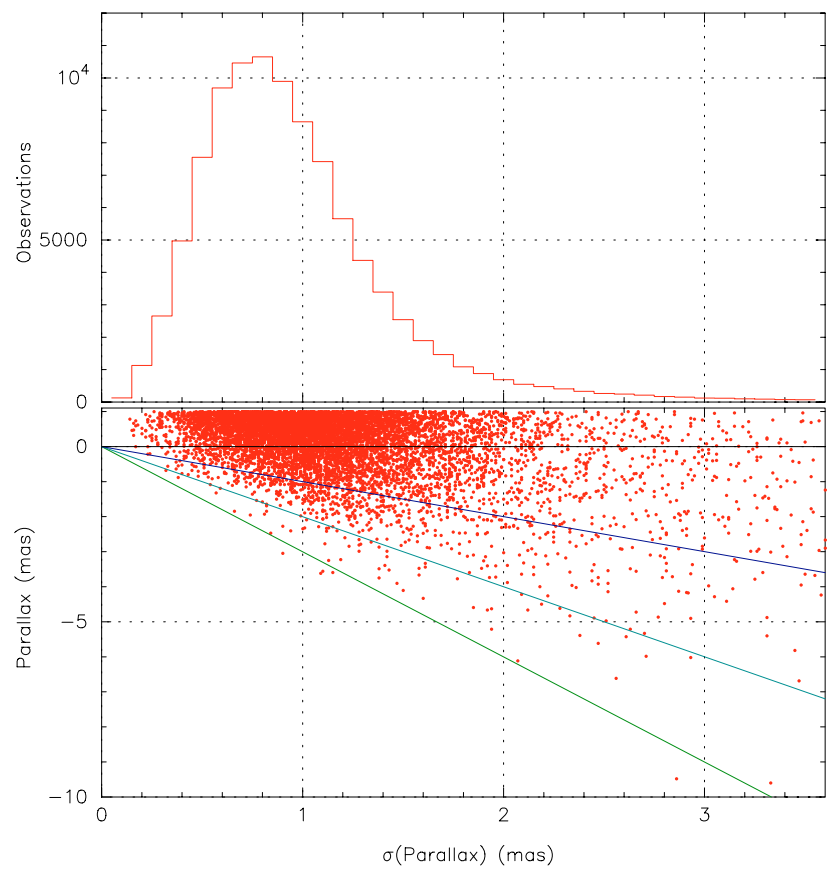

Fig. 16. Top: the histogram, for all single stars with five-parameter solutions, of the formal error on the parallaxes. Bottom: the distribution of parallaxes less than 1 mas s$^{-1}$ as a function of formal error on the parallax determination for the new solution. The diagonal lines show the one, two and three sigma levels as based on the formal errors.

of the numbers of stars in intervals of 0.1 mas width in formal error.

Considering the discussion of the internal errors in the preceding section it seems incorrect to apply any form of error scaling as was done for the 1997 catalogue (see Volume 3 of ESA 1997). What could be considered is a random background noise that can not be detected in the internal error analysis. This could be noise left in the reference frame as determined through the iterations between the along-scan attitude reconstruction and the astrometric-parameter determination. This would most likely be a residual effect still left over from the 1997 catalogue. Such noise residual could be noticeable for those stars with the smallest formal errors. However, among those there is only a very low number of stars with negative parallaxes, far too few for any 


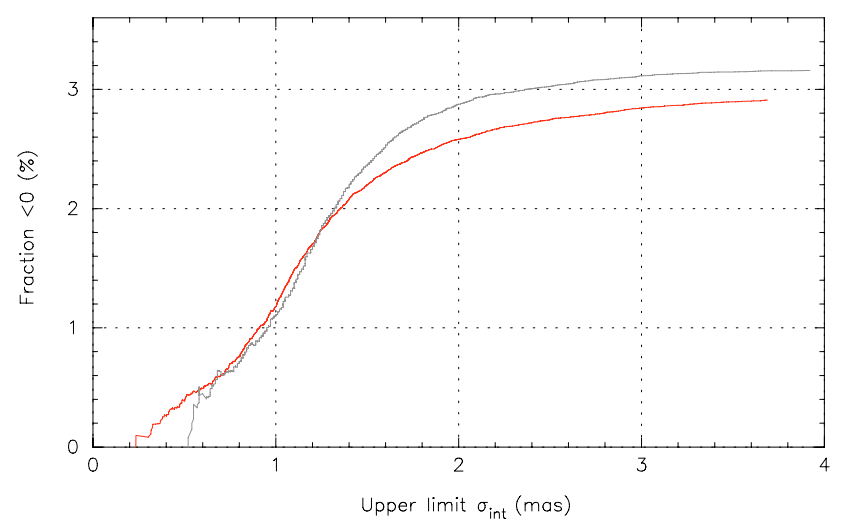

Fig. 17. The cumulative percentage of negative parallaxes as a function of the formal error on the parallax, $\sigma_{\text {int }}$. The grey curve in the background shows the same information as derived from the 1997 catalogue.

Table 2. Data on the ten stars with negative parallaxes and the smallest formal errors.

\begin{tabular}{rrrrrrl}
\hline \hline \multicolumn{1}{c}{ HIP } & $\pi$ & $\sigma_{\pi}$ & $\mathrm{Hp}$ & $B-V$ & \multicolumn{1}{c}{ HD Name } & Spectr. \\
\hline 103312 & -0.22 & 0.23 & 5.801 & 0.408 & 199478 V2140 Cyg B8Ia \\
57741 & -0.20 & 0.24 & 5.761 & 0.233 & 102878 & A3Iab \\
$54751-0.03$ & 0.304 .718 & 0.541 & 97534 V533 Car & A6Ia \\
$44904-0.68$ & 0.32 & 6.861 & 0.150 & 78949 & A1/A2III \\
107749 & -0.21 & 0.32 & 6.569 & 0.381 & 207673 & A2Ib \\
61703 & -0.56 & 0.33 & 6.269 & 0.007 & 109867 KY Mus & B1Ia \\
52004 & -0.16 & 0.33 & 5.551 & 0.500 & 92207 V370 Car & A0Ia \\
97485 & -0.01 & 0.34 & 6.490 & 0.129 & 187459 V1765 Cyg B0.5Ibvar \\
88298 & -0.13 & 0.365 .732 & -0.030 & 164402 & B0Iab... \\
$41074-0.27$ & 0.375 .979 & 0.379 & 70761 & F2Iab \\
\hline
\end{tabular}

objective statistical treatment, as can be seen from Figs. 16 and 17. The overall decrease in the total number of negative parallaxes is one of the clear indications for the improvements achieved with the construction of the new catalogue.

Given the very low numbers of negative parallaxes for those stars with the smallest formal errors, it seems more appropriate to examine the individual cases for which a negative parallax has been obtained rather than to treat these as some statistical variable. The ten stars with negative parallaxes and the smallest formal errors are presented in Table 2. All but one of these stars are supergiants. The spectral information for star HIP 44904 was given by Eggen (1986), who stated that the data for this star could also be interpreted as coming from a high-mass supergiant. The latter seems to be the case, judging by the parallax information. It is also clear from a comparison between colour indices and spectral types that most of these stars are considerably reddened, which should not be surprising given that for most the distance is well over $1 \mathrm{kpc}$.

Unfortunately, there is considerable uncertainty (or intrinsic spread) in the absolute magnitudes of these supergiants (see also Wegner 2007) and the reddening corrections to be applied to the observed magnitudes (Wegner 2003). Also the spectroscopic classification is not always uniquely defined. From an examination of the distribution in parallaxes and apparent magnitudes of the type Ia stars, it seems that the brightest of these supergiants have an absolute magnitude of around -7 , but there could be many fainter by up to three magnitudes (though this is at least partly caused by reddening). There is an indication of some stars still being brighter. These could be the so-called "hypergiants", with absolute magnitudes of around -8 . An example is the star HIP 95657 (HD 183143), identified as a possible hypergiant at

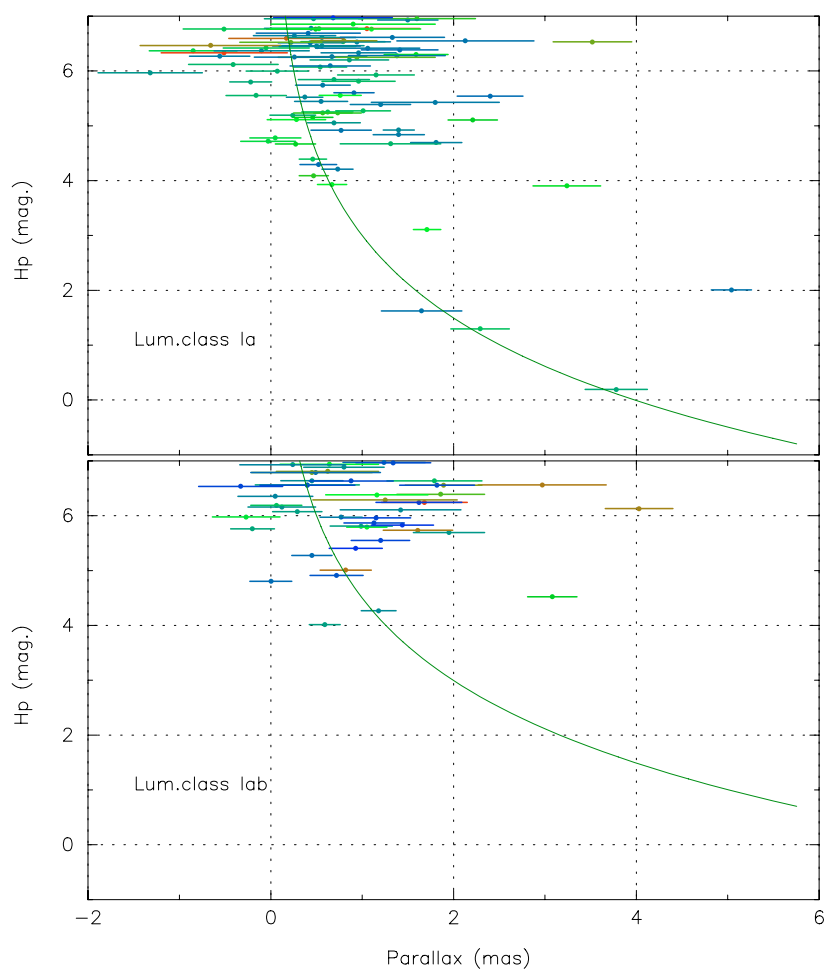

Fig. 18. The distribution of single stars with luminosity class Ia (top) and Iab (bottom) in measured parallax and $\mathrm{Hp}$ magnitude. The magnitudes have not been corrected for reddening. The curve in the upper diagram represents an absolute magnitude of $M_{\mathrm{V}}=-7.0$, and in the lower diagram for $M_{\mathrm{V}}=-5.5$. The observed spread is due to both the formal errors on the parallaxes (as shown) and the (unspecified) formal errors on the spectral classifications.

a distance of $2 \mathrm{kp}$ by Chentsov (2004), for which in the new reduction a parallax of $0.47 \pm 0.54$ has been determined. There are no type Iab stars close enough for a comparable estimate. These results are illustrated by Fig. 18, showing all supergiants with apparent magnitude brighter than $\mathrm{Hp}=7$. Given the uncertainties in classification as well as associated absolute magnitudes and reddening corrections, no firm conclusions should be drawn from the observed distribution of negative parallaxes.

There is, however, another approach that can be made. The distribution of negative parallaxes and their formal errors can be represented as a unit-weight error distribution, assuming all actual parallaxes are zero as an absolute lower limit. In that case the negative parallaxes, divided by their formal errors, represent one half of a Gaussian distribution with $\sigma=1$. The $\chi^{2}$ of the parallax measurements for the ten stars in Table 2 equals 10.34, with 9 degrees of freedom. The $P$-value is 0.32 , so the test gives no ground to reject the assumption that the formal errors are correct. Such procedure can in principle also be extended to a non-zero parallax assumption, but that would have to be based on assumed absolute magnitudes, and would require reddening corrections and the inclusion of all stars with parallaxes smaller than predicted, and not only those with negative parallaxes. This would then make the calculations model dependent. A simplified experiment shows, however, that even if we assume all reference parallaxes to equal 0.2 mas, the $P$-value of the $\chi^{2}$ test would be as high as 0.17 , thus still not inconsistent with the assumptions.

Concluding, it seems from the data that could be analyzed that there is no proof for the presence of an additional noise contribution to the parallax errors. However, the detection capability of the data for such contribution, at a level of a few tenths of a 
Table 3. Comparisons of accumulated weight statistics for parallax measurements of single stars with five-parameter solutions in the old and the new Hipparcos reductions. The errors are given in mas.

\begin{tabular}{rrrrrr}
\hline \hline & \multicolumn{2}{c}{ Observations } & \multicolumn{2}{c}{ Mean error } & Weight \\
Interval & New & Old & New & Old & ratio \\
\hline All & 101783 & 100037 & 0.66 & 0.96 & 2.16 \\
$\mathrm{Hp} \geq 9$ & 34799 & 33347 & 1.23 & 1.33 & 1.23 \\
$\mathrm{Hp}<9$ & 66984 & 66690 & 0.56 & 0.86 & 2.36 \\
$\mathrm{Hp}<7$ & 10883 & 11127 & 0.33 & 0.70 & 4.29 \\
\hline
\end{tabular}

mas, is not very high, and its presence can not be excluded either. Findings for the internal accuracies and the convergence of the new reduction make such noise contributions not very likely.

\section{The potential impact of the new reduction}

The potential impact of the new reduction can be estimated from the formal errors on for example the parallaxes of the single stars. Considering only simple five-parameter solutions, the combined weight for a selection of stars in either the new or the old solution is given by the sum of the inverse squared formal errors:

$w=\sum_{i}\left(1 / \sigma_{\pi}\right)^{2}$.

The change in weight is then given by the ratio of the $w$ values for the new and old reductions. For the entire catalogue, the increase in weight achieved by the new reduction equals a factor 2.16. Details for different magnitude ranges are presented in Table 3. A decrease is noted for the number of bright stars with five-parameter solutions in the new reduction. This is the result of the increased sensitivity at higher accuracy to any orbital disturbance. Somewhat of a surprise has been the improvements observed for the faint stars. However, already the comparisons between the FAST and NDAC data in the 1997 reduction showed that the noise on the data for these stars as published in the old catalogue also contained a significant instrument-modelling component, as was shown by the error correlation statistics (see Volume 3 of ESA 1997).

The largest impact of the new reduction is likely to be for Cepheids and for star clusters and associations. For the Cepheids we examine stars of relatively high intrinsic brightness and at distances above 300 pc. Using the selection criteria for the Cepheid study of Feast \& Catchpole (1997), about four times more stars would be selected. This shows in a study based on the one-but-final iteration results obtained in the construction of the new catalogue by van Leeuwen et al. (2007). While based on the 1997 reduction only the zero point of the Cepheids PL relation could be determined (Feast \& Catchpole 1997), there is now also significant information available on the slope of this relation (see also van Leeuwen 2007).

For open cluster studies preliminary results are presented by van Leeuwen (2007), which show an improvement of the formal errors by about a factor 2 to 2.5 , with formal errors on the parallaxes of the 8 nearest clusters in the range of 0.09 to 0.19 mas. The gains for the open clusters are made in three areas:

- the improvements in the formal errors for the brightest stars;

- the significant reduction in the correlations between the abscissa errors;

- the much improved handling of the connectivity condition in the along-scan attitude reconstruction.

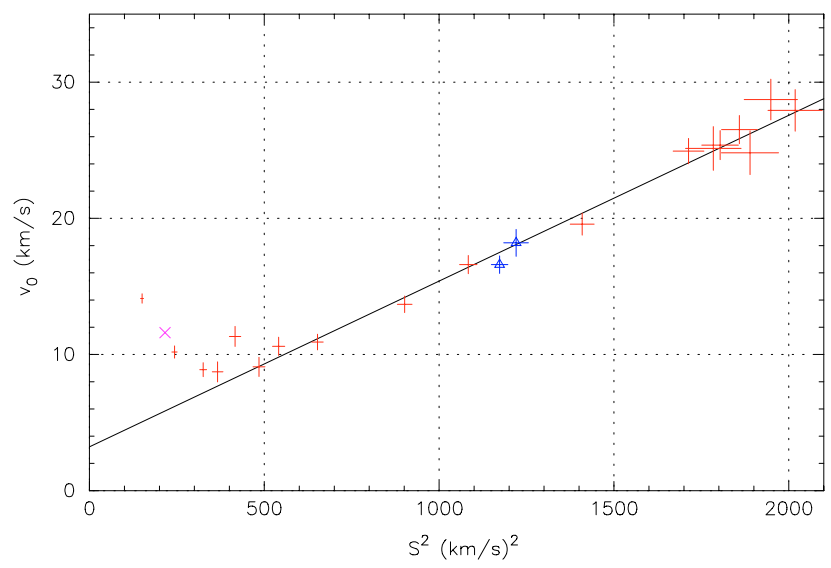

Fig. 19. The asymmetric drift in the galactic rotation shown as the relation between the observed values of $v_{0}$ and $S^{2} \equiv 2 \sigma^{2}$ for main sequence stars and Red Clump and RGB stars (indicated by triangles). The value observed for the Cepheids is shown by $\times$ (from van Leeuwen 2007).

The second point simplifies the reduction of the open cluster data to single cluster parallax and proper motion values considerably. The third point avoids the potential Pleiades problem to which open cluster data in particular can be very sensitive. This is due to the concentrations of relatively bright stars and the weight these may potentially assert in the along-scan attitude reconstruction. The open cluster analysis and results will be presented in a separate paper, currently in preparation.

A significant impact can also be made on studies of galactic dynamics. A repeat of the study by Binney et al. (2000), for example, shows a factor two more stars available, resulting in additional measurements towards later spectral types (van Leeuwen 2007). Three more data points could be added to the asymmetric drift diagram, for Cepheids, Red Clump and RGB stars, as is shown in Fig. 19. Preliminary results on the solar motion and galactic rotation, as based on the new reduction, can also be found in van Leeuwen (2007).

\section{Conclusions}

The possibilities to verify astrometric data down to a level of 0.1 mas are rather limited, in particular for relatively bright stars. The internal verification has to provide the main body of evidence, as almost no data is available for an independent external verification. Independent here means free from assumptions derived from the application of those data to the analysis of astrophysical objects, and in particular when that involves assumed absolute magnitudes and their intrinsic dispersion. The internal verification of the new reduction appears to provide fully selfconsistent results and, as such, provides the main element of the data quality verification. The limitations of the external verification leave open a number of questions, for which the answers may not be available until a much improved independent catalogue, such as the one expected from the Gaia mission, is available.

The improvements achieved by the new reduction of the Hipparcos astrometric data put the results of this mission significantly above its original aim, which was set at 2 mas. In setting this aim, the efficiency of the detectors had been underestimated, and the noise contribution of the instrument calibrations overestimated. The original reductions, as published in 1997, already provided data that was a factor two better on average. The new reduction has made this a factor three on average, and more than 
a factor ten for many of the brightest stars. With these improvements, significant progress can again be made in a wide range of luminosity calibration studies and studies of galactic dynamics.

Above all, however, the new reduction has shown that the principle of Hipparcos for obtaining absolute parallaxes works, though it requires very careful implementation of various aspects of the data processing, and in particular the along-scan attitude reconstruction. The latter could only be achieved for the Hipparcos mission through a thorough understanding of the satellite dynamics.

Acknowledgements. I would like to express my thanks to Lennart Lindegren, Dafydd W. Evans, Rudolf Le Poole and Elena Fantino for their support, suggestions, ideas and corrections along the long way of this study.

\section{References}

Arenou, F., Lindegren, L., Frœschlé, M., et al. 1995, A\&A, 304, 52

Arias, E. F., Charlot, P., Feissel, M., \& Lestrade, J.-F. 1995, A\&A, 303, 604

Binney, J., Dehnen, W., \& Bertelli, G. 2000, MNRAS, 318, 658

Chentsov, E. L. 2004, Astron. Lett., 30, 325

Eggen, O. J. 1986, PASP, 98, 423

ESA 1992, The Hipparcos Input Catalogue, SP 1136 (ESA)

ESA 1997, The Hipparcos and Tycho Catalogues, SP 1200 (ESA)

Feast, M. W., \& Catchpole, R. M. 1997, MNRAS, 286, L1

Feissel, M., \& Mignard, F. 1998, A\&A, 331, L33

Knapp, G., Pourbaix, D., \& Jorissen, A. 2001, A\&A, 371, 222
Knapp, G. R., Pourbaix, D., Platais, I., \& Jorissen, A. 2003, A\&A, 403, 993 Kovalevsky, J., Falin, J. L., Pieplu, J. L., et al. 1992, A\&A, 258, 7 Lestrade, J.-F., Jones, D. L., Preston, R. A., et al. 1995, A\&A, 304, 182 Lestrade, J.-F., Preston, R. A., Jones, D. L., et al. 1999, A\&A, 344, 1014 Lindegren, L. 1995, A\&A, 304, 61

Lindegren, L. 2005, in The Three-Dimensional Universe with Gaia, ed. C. Turon, K. S. O'Flaherty, \& M. A. C. Perryman, ESA SP-576, 29

Lindegren, L., Hoeg, E., van Leeuwen, F., et al. 1992, A\&A, 258, 18 Makarov, V. 2002, AJ, 124, 3299

Narayanan, V. K., \& Gould, A. 1999, ApJ, 523, 328

O’Mullane, W., Lammers, U., Bailer-Jones, C., et al. 2006, ArXiv Astrophysics e-prints

Platais, I., Pourbaix, D., Jorissen, A., et al. 2003, A\&A, 397, 997

Soderblom, D. R., Nelan, E., Benedict, G. F., et al. 2005, AJ, 129, 1616

Turon, C., Gomez, A., Crifo, F., et al. 1992, A\&A, 258, 74

van der Marel, H. 1988, Ph.D. Thesis, Technische Universiteit Delft

van der Marel, H., \& Petersen, C. S. 1992, A\&A, 258, 60

van Leeuwen, F. 1999, in Harmonizing cosmic distance scales in a postHipparcos era, ed. D. Egret, \& A. Heck (PASPC), 167, 52

van Leeuwen, F. 2005, A\&A, 439, 805

van Leeuwen, F. 2007, Hipparcos, the new reduction of the raw data (Dordrecht: Springer)

van Leeuwen, F., \& Evans, D. W. 1998, A\&A, 323, 157

van Leeuwen, F., \& Fantino, E. 2003, Space Sci. Rev., 108, 537

van Leeuwen, F., \& Fantino, E. 2005, A\&A, 439, 791

van Leeuwen, F., Feast, M. W., Whitelock, P. A., \& Laney, C. D. 2007, ArXiv e-prints, 705

Wegner, W. 2003, Astron. Nachr., 324, 219

Wegner, W. 2007, MNRAS, 374, 1549 